\title{
Métodos de Pontos Interiores Aplicados ao Problema de Pré-Despacho do Sistema Hidroelétrico com Manobras e Reserva Girante
}

\author{
S.M.S. CARVALHO ${ }^{*}$, M.V. COELHO ${ }^{2}$ e A.R.L. OLIVEIRA ${ }^{3}$
}

Recebido em 19 setembro 2016 / Aceito em 14 janeiro 2017

RESUMO. Nesse artigo os métodos de pontos interiores primais-duais foram desenvolvidos para o problema de minimização das perdas na geração e transmissão do pré-despacho DC de um sistema de potência hidroelétrico onde ocorrem adicionalmente manobras programadas e reserva girante. São abordados tanto o aspecto do número de iterações para convergência do método quanto a eficiência da resolução dos sistemas lineares envolvidos para obtenção de iterações rápidas.

Palavras-chave: pontos interiores, pré-despacho, programação linear.

\section{INTRODUÇÃO}

No pré-despacho de sistemas hidroelétricos, cada usina deve cumprir uma meta para um determinado dia, estabelecida pelo planejamento de longo prazo. No entanto, com a variação da demanda e possíveis contingências ao longo do dia, se faz necessário o uso de serviços ancilares, ou seja, serviços que contribuem para segurança, confiabilidade e qualidade do suprimento de energia elétrica como por exemplo, manobras programadas e reserva girante.

A consideração destes serviços ancilares adicionais, aproxima ainda mais o modelo do problema de pré-despacho ao sistema brasileiro de acordo com o Operador Nacional do Sistema.

A contribuição deste trabalho é a implementação dos métodos de pontos interiores para o prédespacho contendo estes serviços ancilares e o estudo comparativo com o problema sem tais restrições, já estudado em $[14,10,11]$, no que diz respeito a qualidade da solução, custo operacional e a eficiência computacional.

\footnotetext{
*Autor correspondente: Silvia Maria Simões Carvalho

${ }^{1}$ Departamento de Física, Química e Matemática - DFQM - UFSCar - Universidade Federal de São Carlos, 18052-780 Sorocaba, SP, Brasil. E-mail: silviamsc@ufscar.br

2 Instituto de Ciência e Tecnologia - ICT - UNIFAL-MG - Universidade Federal de Alfenas, Rodovia José Aurélio Vilela, 11999 (BR 267 - km 533), Cidade Universitária, 37715-400 Poços de Caldas, MG, Brasil.

E-mail: mayk.coelho@unifal-mg.edu.br

${ }^{3}$ Instituto de Matemática, Estatística e Computação Científica - IMECC - UNICAMP, R. Sérgio Buarque de Holanda, 651, Cidade Universitária, 13083-859 Campinas, SP, Brasil. E-mail: aurelio@ime.unicamp.br
} 
Nas Seções 2 e 3 são discutidos respectivamente os serviços ancilares de manobras programadas e os de reserva girante. Na Seção 4 é apresentada a formulação do problema de pré-despacho com a aplicação de manobras e reservas. A Seção 5 são feitos estudos de casos no sistema IEEE 30 barras e discutidos os resultados computacionais. Por fim, na Seção 6 são apresentadas as conclusões obtidas com este trabalho.

\section{MANOBRAS PROGRAMADAS}

O uso de manobras programadas se dá pela necessidade de manutenção em geradores e/ou em linhas de transmissão para adaptar o sistema à nova carga, ou mesmo devido a interrupções momentâneas inesperadas. Este tipo de serviço possibilita atender a demanda com eficiência e de modo estável.

A sequencia de operações na rede deve obedecer requisitos elétricos e critérios previamente estabelecidos, normatizados por órgãos governamentais ou pela concessionária de transmissão ou distribuição de energia elétrica [3]. O objetivo é atender a todas as cargas, sem sobrecarregar linhas, alimentadores e usinas de geração.

Este serviço modifica a topologia ativa da rede, necessitando que um novo despacho seja realizado, visto que há um desligamento de linhas de transmissão em determinados períodos do dia. Obter uma nova política de despacho para estes períodos de manobra é de extrema importância, não apenas para que o serviço seja realizado, mas também para manter o sistema carga-geração em equilíbrio. Normalmente, são realizadas de quatro a seis manobras por dia $^{4}$.

\subsection{Modelagem das Manobras}

Neste trabalho são considerados três tipos de manobras:

- Manobras de Linhas - Quando um ramo é retirado do sistema;

- Manobras de Barras - Quando geradores ou cargas são desligados do sistema;

- Manobras Simultâneas de Linhas e Barras.

Todas estas manobras provocam uma alteração na topologia da rede, deste modo, é preciso um estudo na modelagem do problema para entender quais alterações devem ser realizadas. Em uma matriz de incidência da rede, cada coluna se refere a uma linha de transmissão, ou ramo. Deste modo, algebricamente uma manobra de linhas significa a retirada de colunas da matriz de incidência. Esta alteração implica também na retirada de linhas da matriz de reatância referentes a estes ramos [2].

Para fins de implementação, não é preciso armazenar tantas matrizes de incidência e reatância quanto o número de manobras, basta apenas armazenar o índice das colunas e linhas, respectivamente, referentes aos ramos que saem e as horas em que tais manobras serão realizadas.

\footnotetext{
${ }^{4}$ Segundo procedimentos do ONS - Operador Nacional do Sistema
} 
Deste modo, a cada hora utiliza-se apenas as colunas e linhas ativas destas matrizes e dos vetores multiplicados por elas.

Estas manobras de linhas podem resultar em redes desconectadas, gerando um apagão setorial, porém, pode ser evitado utilizando heurísticas para construção de árvore geradora de modo que todas as manobras possam ser realizadas apenas nos ramos adicionais [9]. Dependendo da topologia da rede, pode haver ramos que não possam ser manobrados, nestes casos, sugere-se a instalação de linhas adicionais.

Em manobras de barras, as cargas têm variações nem sempre previsíveis com o tempo, fator que pode dificultar a modelagem do sistema. Diferentemente das manobras de linhas, não se pode optar por ramos pertencentes ou não a árvore geradora, pois obviamente ao desligar uma barra, linhas da árvore geradora e de seus arcos adicionais devem ser desligados. Assim como antes, caso resulte em redes desconectadas, sugere-se o mesmo procedimento.

Neste trabalho escolheu-se fazer manobras somente nas barras com grau menor que dois (apenas um arco da árvore o incide). Alterar a heurística para obter árvores com essas características é relativamente simples. Supõe-se que $n$-arcos incidem na barra a ser manobrada, $\log 0 n$-linhas e $n$-colunas das matrizes de reatância e de incidência, respectivamente, devem ser retirados.

\section{RESERVA GIRANTE}

Quando um sistema de potência sofre uma contingência, como a perda de unidades de geração e/ou de transmissão, podem ocorrer desequilíbrios no conjunto carga-geração ou extrapolações nos limites de capacidade dos circuitos de transmissão. Nessas situações torna-se necessário o emprego de medidas corretivas que eliminem estas violações operativas, reconduzindo o sistema a um ponto de operação seguro, dentre os quais destaca-se o re-despacho da potência ativa, fazendo uso de uma reserva girante de potência em determinadas unidades de geração.

O serviço ancilar de reserva de potência é provido por geradores conectados à rede elétrica e sincronizados com o sistema de potência. Este serviço ancilar visa disponibilizar uma quantidade extra de potência ativa, que pode ser imediatamente utilizada durante uma situação de contingência para restabelecer o equilíbrio no conjunto carga-geração.

Para que esta reserva seja disponibilizada, torna-se necessário que determinados geradores reduzam a geração de potência ativa durante a operação normal do sistema, mantendo uma parcela de sua capacidade de produção para situações de contingência, mas ainda atendendo ao mercado energético $[1,7]$.

A quantidade da reserva girante disponível depende do nível de confiabilidade e de segurança que se deseja, como por exemplo, pode-se exigir uma reserva girante que vise suprir a queda da maior unidade geradora do sistema. No Brasil, o OIS (Operador Independente do Sistema) pode requisitar, para garantir uma operação eficiente e segura do sistema, o re-despacho de um gerador, reduzindo sua potência ativa a fim de permitir que o mesmo forneça mais reserva girante [12]. 
A reserva girante é um produto fundamentalmente diferente da energia. Enquanto que para a energia negociada sua utilização é programada antecipadamente, a reserva girante deve estar disponível para ser usada imediatamente caso ocorra uma contingência no sistema.

Contudo, é possível que a receita de venda de energia destes geradores sofra uma queda devido à esta redução de geração, implicando em um custo para prover o serviço ancilar [5, 8, 13, 6], que deve ser remunerado de alguma forma, porém, este estudo não será abordado neste trabalho.

\subsection{Modelagem da Reserva Girante}

Para o gerenciamento da reserva girante são necessárias três restrições:

- Limitar o quanto cada gerador pode reservar de sua potência;

- Garantir que a potência fornecida para o sistema somada com a reservada seja igual a capacidade máxima do gerador;

- Garantir que toda potência reservada por estes geradores seja maior ou igual ao exigido pelo CAG (Controle Automático de Geração).

Estas três restrições podem ser modeladas da seguinte forma [4]:

$$
\begin{gathered}
0 \leq r_{e} \leq r_{e u} \\
p+r_{e}=p_{u} \\
v^{t} r_{e} \geq \tau .
\end{gathered}
$$

Onde $r_{e}$ e $r_{e u}$ são o vetor de reserva girante e de máxima reserva ${ }^{5}$ respectivamente, $p$ e $p_{u}$ são o vetor de potência gerada e o de máxima geração respectivamente, $\tau$ o mínimo de reserva girante exigida pela CAG e $v$ é um vetor de binário, sendo 1 nas componentes relacionadas aos geradores com política de reserva.

\section{O PROBLEMA DE PRÉ-DESPACHO COM MANOBRAS E RESERVA GIRANTE}

O problema de pré-despacho de um sistema elétrico é operacional e de curto-prazo, que pode ser formulado com a minimização de uma função quadrática com variáveis separáveis que representa as perdas na transmissão e os custos de geração. As leis de Kirchoff caracterizam restrições do problema.

O custo de geração associado às termoelétricas também é uma função quadrática independente para cada gerador. Vale ressaltar que os métodos de pontos interiores para problemas com esta característica apresentam desempenho similar ao obtido para problemas lineares. Em particular o esforço por iteração é virtualmente o mesmo em ambas as situações $[15,16]$.

\footnotetext{
${ }^{5}$ Para que a unidade geradora mantenha-se ligada para o fornecimento imediato de sua potência reservada, $r_{e u}$ deve ser inferior a $p_{u}$.
} 
O problema de Pré-despacho como descrito é apresentado em [14]. Ao inserir o serviço de manobras de linhas e de barras neste problema, tem-se um conjunto de equações matriciais que se modificam ao longo de horizontes de estudo, em consequência das retiradas de colunas e linhas citadas anteriormente. Além disso, inserindo as equações (3.1), (3.2) e (3.3) temos o seguinte problema modelado:

\subsection{Modelagem do Problema}

Será apresentado agora o modelo de fluxo de potência ótimo com manobras programadas e reserva girante na forma padrão.

$$
\text { Min. } \begin{aligned}
\varphi(f, p) & \\
\qquad . a \quad B^{k} f^{k}-E^{k} p^{k} & =d^{k} \\
p^{k}+r_{e}^{k} & =p_{u} \\
v^{t} r_{e}^{k}-s^{k} & =\tau^{k} \\
f^{k}+s_{f}^{k} & =f_{u} \\
p^{k}+s_{p}^{k} & =p_{u} \\
r_{e}^{k}+s_{r_{e}}^{k} & =r_{e u} \\
\sum_{k=1}^{24} p^{k} & =q \\
\forall k=1, \ldots, t . &
\end{aligned}
$$

- $\varphi(f, p)=\frac{\alpha}{2} \sum_{k=1}^{24}\left[\left(f^{k}\right)^{t} R^{k} f^{k}+c_{f}^{t} f^{k}\right]+\frac{\beta}{2} \sum_{k=1}^{24}\left[\frac{1}{2}\left(p^{k}\right)^{t} Q^{k}\left(p^{k}\right)+c_{p}^{t} p^{k}\right]$;

- $f \in R^{n \times 1}$ representa o fluxo de potência ativa;

- $p \in R^{g \times 1}$ representa a geração de potência ativa;

- $Q \in R^{g \times g}$ representa a componente quadrática do custo de geração;

- $R \in R^{n \times n}$ representa a matriz diagonal de resistência das linhas;

- $d \in R^{m \times 1}$ representa a demanda de potência ativa;

- $X \in R^{n-m+1 \times n}$ representa a matriz de reatância das linhas;

- $E$ representa uma matriz de ordem $m \times g$ com cada coluna contendo exatamente um elemento igual a 1 e os demais elementos nulos; 
- $A \in R^{m \times n}$ representa a matriz de incidência da rede de transmissão;

- $c_{p} \in R^{g \times 1}$ representa a componente linear do custo de geração;

- $c_{f} \in R^{n \times 1}$ representa a componente linear do custo de transmissão;

- $f_{u} \in R^{n \times 1}, p_{u} \in R^{g \times 1}$ representam o limite de fluxo e geração de potência ativa respectivamente;

- $\alpha$ e $\beta$ são ponderações dos objetivos a minimizar;

- $q \in R^{g \times 1}$ representa a meta de geração de energia estabelecida pelo planejamento a longoprazo;

- $r_{e}$ e $r_{e u} \in R^{g \times 1}$ representam respectivamente a reserva de potência ativa e o máximo de reserva;

- $\tau$ representa o mínimo de reserva de potência exigida em um subconjunto $\Gamma$ de geradores;

- $v \in R^{g \times 1}$ é um vetor tal que $v_{i}= \begin{cases}1 & \text { se o gerador } i \in \Gamma \\ 0 & \text { caso contrário. }\end{cases}$

As matrizes $B=[A ; X]$ e $E$ variam de dimensão, conforme as modificações na rede provocadas pelas manobras realizadas ao longo de horizonte de estudos.

Note que a inclusão de reserva girante torna redundante o limite superior da capacidade de geração das usinas que participam da reserva.

\subsection{Aplicando Métodos de Pontos Interiores ao Modelo}

O problema dual de 4.1 é escrito da seguinte forma:

$$
\begin{aligned}
& \operatorname{Max} . \quad \phi\left(y, y_{r}, w, w_{p}, w_{r e}\right)-\varphi(f, p) \\
& \text { s.a } \quad\left(B^{t}\right)^{k} y^{k}-w_{f}^{k}-R^{k} f^{k}+z_{f}^{k}=c_{f} \\
& -E^{t} y^{k}+y_{r}^{k}-w_{p}^{k}+y_{a}-Q^{k} p^{k}+z_{p}^{k}=c_{p} \\
& y_{r}^{k}+w^{k} \tau-w_{r e}^{k}+z_{r e}^{k} \quad=0 \\
& \left(w^{k}, w_{f}^{k}, w_{p}^{k}, w_{r e}^{k}, z_{f}^{k}, z_{p}^{k}, z_{r e}^{k}\right) \quad \geq 0 \\
& \left(y_{f}, y_{a}\right) \quad \text { livre. }
\end{aligned}
$$

onde

$$
\phi\left(y, y_{r}, w, w_{p}, w_{r e}\right)=\left(d^{k}\right)^{t} y^{k}+p_{u}^{t} y_{r}^{k}+\tau^{t} w^{k}-p_{u}^{t} w_{p}^{k}-r_{e u}^{t} w_{r e}+q^{t} y_{a} .
$$


Desta maneira, obtêm-se as seguintes condições de otimalidade:

Factibilidade Primal

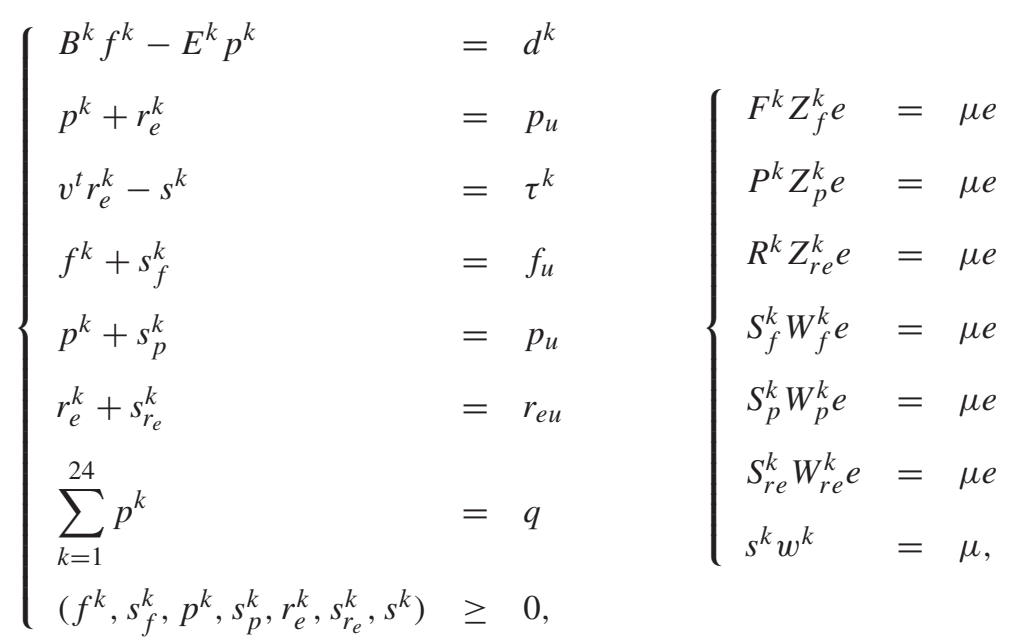

Factibilidade Dual

$$
\begin{cases}\left(B^{t}\right)^{k} y^{k}-w_{f}^{k}-R^{k} f^{k}+z_{f}^{k} & =c_{f} \\ -E^{t} y^{k}+y_{r}^{k}-w_{p}^{k}+y_{a}-Q^{k} p^{k}+z_{p}^{k} & =c_{p} \\ y_{r}^{k}+w^{k} \tau-w_{r e}^{k}+z_{r e}^{k} & =0 \\ \left(w^{k}, w_{f}^{k}, w_{p}^{k}, w_{r e}^{k}, z_{f}^{k}, z_{p}^{k}, z_{r e}^{k}\right) & \geq 0 \\ y_{f}^{k}, y_{a}^{k} \text { livres. } & \end{cases}
$$


Aplicando o método de Newton às condições de otimalidade do problema tem-se o seguinte sistema:

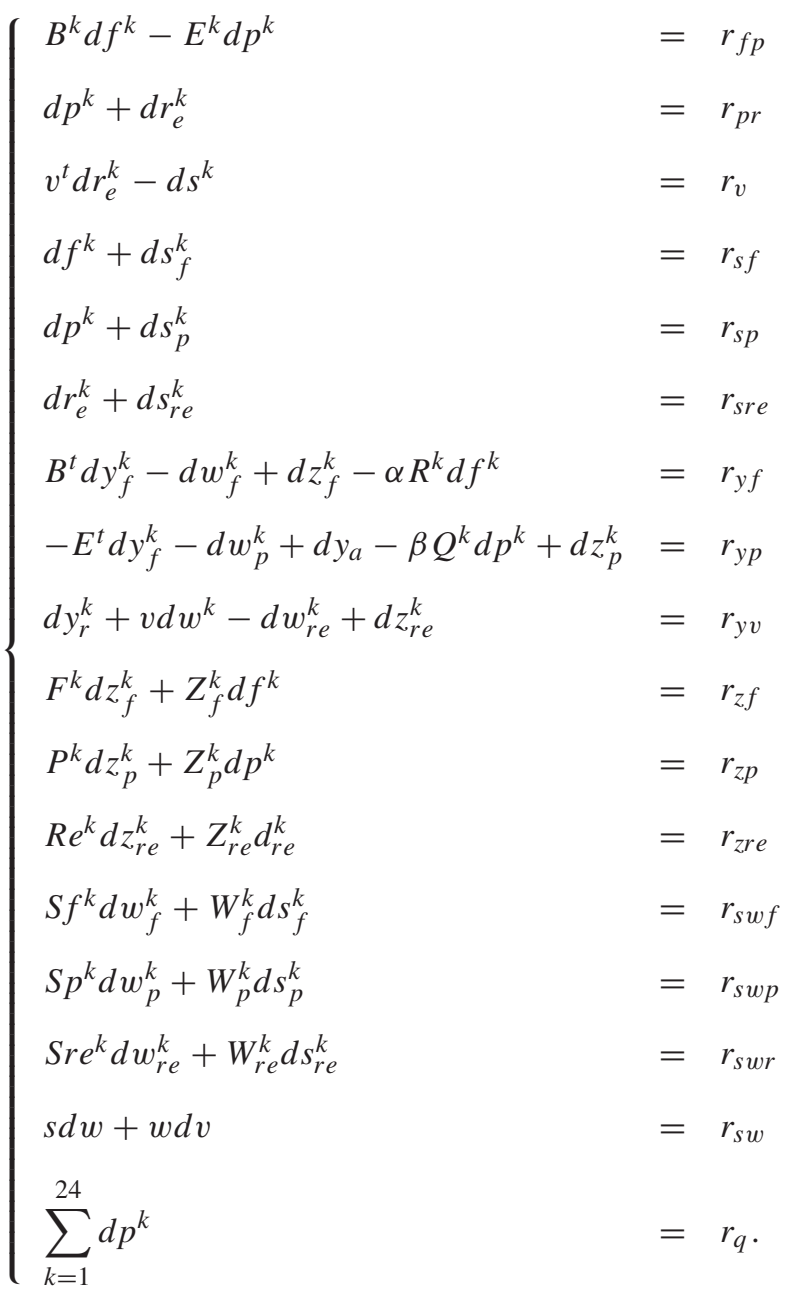

O sistema 4.3 pode ser reduzido via retro-substituições de variáveis. Deste modo, pode-se iniciar este processo isolando as direções referentes às variáveis duais não livres e as primais de folga. Substituindo-as nas equações restantes do sistema 4.3 tem-se o seguinte:

$$
\left\{\begin{array}{l}
\sum_{D^{k} d y_{r}^{k}}=\bar{r}_{q}^{k} \\
s d w+w d s=r_{s w} .
\end{array}\right.
$$

onde,

$$
\begin{aligned}
D^{k} & =M^{k}+\left(s^{k}\right)^{-1} w^{k}\left(D_{r e}^{k}\right)^{-1} v\left(D_{w}^{k}\right)^{-1} v^{k}\left(D_{r e}^{k}\right)^{-1} \\
M^{k} & =\left(D_{p}^{k}\right)^{-1}-D y_{r}^{k} \\
\bar{r}_{q}^{k} & =r_{q}^{k}-\sum\left[\bar{r}_{p r}^{k}-\left(s^{k}\right)^{-1}\left(D_{r e}^{k}\right)^{-1} v D_{w}^{-1}\left(r_{s w}^{k}+w^{k} v^{t}\left(D_{r e}^{k}\right)^{-1} r_{y v}^{k}+w^{k} r_{v}^{k}\right)\right] .
\end{aligned}
$$




\section{ESTUDO DE CASOS}

Foram realizados testes computacionais com as seguintes configurações: Notebook Dell Latitude 3470 com Windows 10 8GB RAM com processador Intel ${ }^{\circledR}$ Core(MT) i7-6500U (2 núcleos e 4 Processadores), CPU 2,50GHz, com buster de 3,0GHz.

Utilizando método de pontos interiores primal-dual, os experimentos computacionais a seguir foram feitos variando o número de manobras diárias em até no máximo cinco, simulando o procedimento usual no sistema elétrico brasileiro.

Os testes realizados nesta seção partiram do pontos iniciais conforme definido em [11], por apresentarem bons resultados em experimentos anteriores.

Um estudo ilustrativo foi realizado com sistema de 30 barras do Institute of Electrical and Electronics Engineers IEEE30 representando o meio-oeste americano, com 6 unidades geradoras, potência instalada de 600MW (100MW cada) com custos iguais. Para fim de testes, foi definido fluxo máximo de 100MW em cada linha de transmissão.

As ponderações da função objetivo foram consideradas como $\alpha=C M$ e $\beta=1$, onde CM é o custo marginal dos geradores obtido $\operatorname{com} \alpha=1$ e $\beta=0$.

Para manobras de linhas foram considerados os ramos 1-3, 9-10, 27-28, 5-7, e 8-28. Estes ramos são os de maior fluxo fora da árvore geradora no despacho sem manobras. Foram realizados dois tipos de manobras, uma desligando todos no intervalo entre 17 e 23 horas (dados Tabela 1) e outra nos intervalos [1-4], [6-9], [11-15], [17-20] e [21-23] respectivamente (dados Tabela 2).

Para manobras de barras, foram realizados testes com o gerador 2 sendo desligado entre 5 e 7 horas da manhã, considerando variação de manobras de linhas no intervalo de 17 às 23 horas (dados Tabela 3).

Para reserva girante foram realizados testes com o conjunto de geradores 5 e 13 e com o conjunto 5 e 8. Para ambos conjuntos foi exigido uma reserva girante de 90MW. Esta definição de reserva é suficiente caso haja uma contingência que envolva o desligamento de um gerador.

O Gráfico na Figura 1(a) representa o despacho de potência sem manobras e sem reserva.

Com os dados das Tabelas 1 e 2 é possível observar que manobras programadas não influenciam no número de iterações, resultando apenas em um pequeno acréscimo no tempo computacional. O despacho de potência não se altera, considerando apenas manobras de linhas.

Ainda sem considerar reserva girante, é possível observar que o tempo computacional de operação com manobras simultâneas é levemente inferior ao com manobras intercaladas. Esta leve alteração se dá devido ao uso de mais de duas topologias da rede para representar a retirada de cada ramo ao longo da operação.

Quando o fluxo nos ramos suporta a produção do gerador a possibilidade de manobras nos demais ramos fora da arvore aumenta. Caso o fluxo nos ramos seja reduzido para 50MW manobras nos ramos 1-3 e 9-7 são inviáveis, congestionando a rede. 
Tabela 1: Reserva Girante e Manobras de Linhas simultâneas entre 15 e 23 horas.

\begin{tabular}{|c|c|c|c|c|c|c|}
\hline & \multicolumn{2}{|c|}{ Sem Reserva } & \multicolumn{2}{c|}{ Com Reserva (5-13) } & \multicolumn{2}{c|}{ Com Reserva (5-8) } \\
\hline Manobras & Iterações & Tempo(s) & Iterações & Tempo(s) & Iterações & Tempo(s) \\
\hline 0 & 3 & 0,0964 & 4 & 0,2628 & 4 & 0,1235 \\
1 & 3 & 0,1015 & 5 & 0,3281 & 4 & 0,1248 \\
2 & 3 & 0,1009 & 5 & 0,3302 & 4 & 0,1276 \\
3 & 3 & 0,1061 & 5 & 0,3332 & 4 & 0,1328 \\
4 & 3 & 0,1086 & 5 & 0,3451 & 4 & 0,1338 \\
5 & 3 & 0,1045 & 5 & 0,3462 & 4 & 0,1372 \\
\hline
\end{tabular}

Tabela 2: Reserva Girante e Manobras de Linhas ao longo do dia em horários distintos.

\begin{tabular}{|c|c|c|c|c|c|c|}
\hline & \multicolumn{2}{|c|}{ Sem Reserva } & \multicolumn{2}{c|}{ Com Reserva (5-13) } & \multicolumn{2}{c|}{ Com Reserva (5-8) } \\
\hline Manobras & Iterações & Tempo(s) & Iterações & Tempo(s) & Iterações & Tempo(s) \\
\hline 0 & 3 & 0,0964 & 4 & 0,2641 & 4 & 0,1231 \\
1 & 3 & 0,1195 & 4 & 0,2217 & 4 & 0,1318 \\
2 & 3 & 0,1101 & 4 & 0,2703 & 4 & 0,1452 \\
3 & 3 & 0,1144 & 4 & 0,2839 & 4 & 0,1536 \\
4 & 3 & 0,1225 & 5 & 0,3671 & 4 & 0,1644 \\
5 & 3 & 0,1279 & 5 & 0,3818 & 4 & 0,1739 \\
\hline
\end{tabular}

Tabela 3: Manobra de Barra Gerador 2 e de Linhas com Reserva Girante.

\begin{tabular}{|c|c|c|c|c|c|c|}
\hline & \multicolumn{2}{|c|}{ Sem Reserva } & \multicolumn{2}{c|}{ Com Reserva (5-13) } & \multicolumn{2}{c|}{ Com Reserva (5-8) } \\
\hline Manobras & Iterações & Tempo(s) & Iterações & Tempo(s) & Iterações & Tempo(s) \\
\hline 0 & 5 & 0.1527 & 7 & 0,3453 & 7 & 0,2146 \\
1 & 5 & 0,1668 & 9 & 0,4765 & 7 & 0,2215 \\
2 & 5 & 0,1493 & 9 & 0,4358 & 7 & 0,2193 \\
3 & 5 & 0,1438 & 9 & 0,4305 & 7 & 0,2173 \\
4 & 5 & 0,1416 & 9 & 0,4182 & 7 & 0,2058 \\
5 & 5 & 0,1561 & 9 & 0,4326 & 7 & 0,2157 \\
\hline
\end{tabular}

Considerando o uso de reserva girante é possível notar um esforço maior para obter o despacho ótimo devido o aumento do número de iterações e consequentemente do tempo computacional. Porém, como a demanda para o conjunto de geradores 5 e 13 é maior que a demanda para o conjunto 5 e 8 , o despacho para o segundo é obtido com menos iterações.

É possível observar a influência da reserva girante no despacho obtido analisando os gráficos nas Figuras 1(c) e 1(d) e comparando com o da Figura 1(a). 
Para o conjunto 5 e 13 foi necessário reduzir a produção de ambos a partir das 7 horas de modo que a reserva do conjunto chegasse a 90MW, forçando os demais geradores a aumentarem sua produção. Já para o conjunto 5 e 8, a reserva no segundo gerador já era alta nas primeiras 18 horas, provocando uma leve diminuição na produção do conjunto apenas entre 18 e 21 horas, justificando o número menor de iterações.

Na Tabela 3 tem-se os dados obtidos para as manobras de barra. É possível observar nesta tabela que o desligamento do gerador 2 faz com que a obtenção de um despacho ótimo se torne mais trabalhoso, aumentando o número de iterações em todos os testes.

O gráfico na Figura 1(b) representa o despacho com a manobra no gerador 2 sem considerar reserva girante. É possível notar um leve acréscimo de produção nos demais horários devido este desligamento de uma hora. Os despachos com reserva foram semelhantes aos obtidos anteriormente, inserindo apenas a parada do gerador 2 às 6 horas e com uma leve translação para cima nos demais horários.

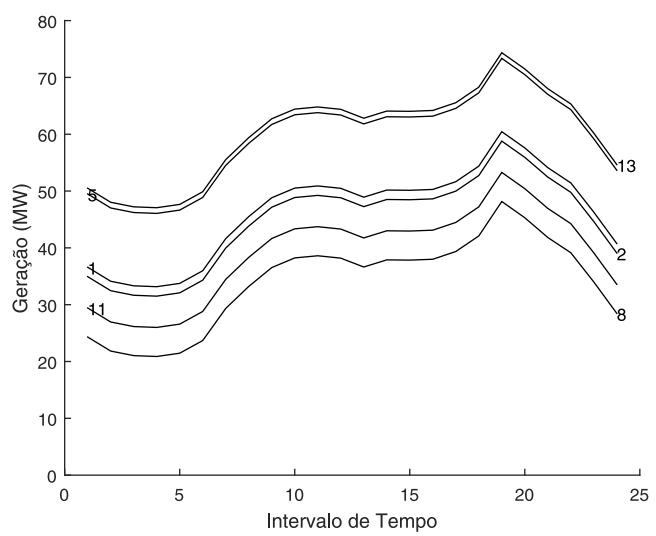

(a) Despacho sem Manobras e sem Reserva Girante.

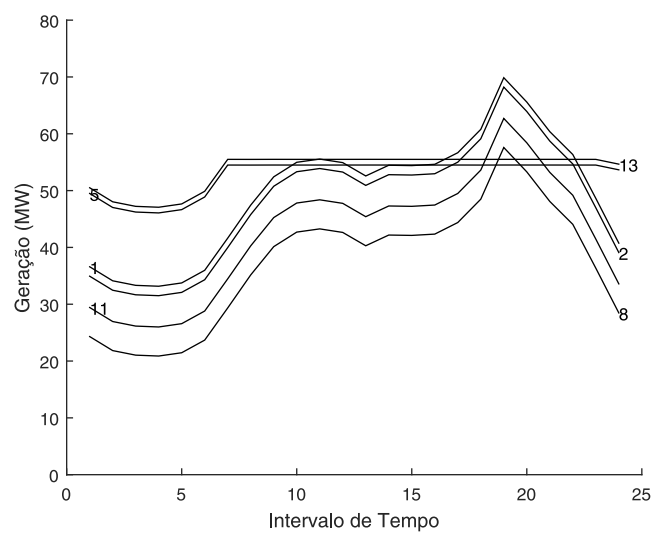

(c) Despachos com Reserva envolvendo os geradores 5 e 13.

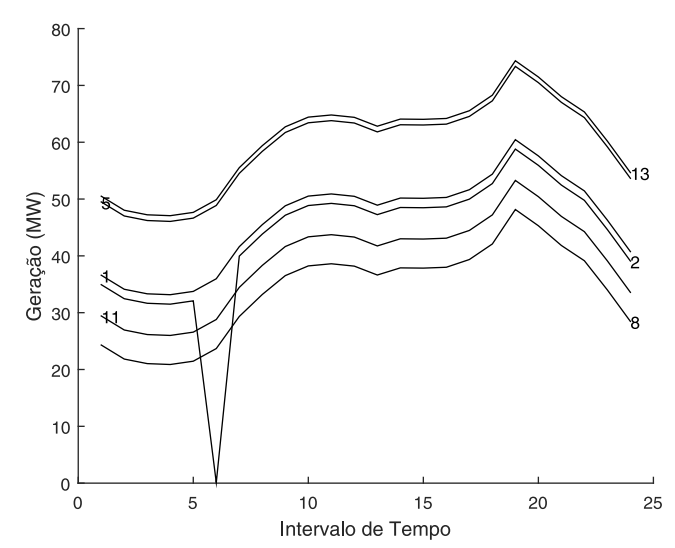

(b) Despacho com Manobra na Barra 2 às 6 horas.

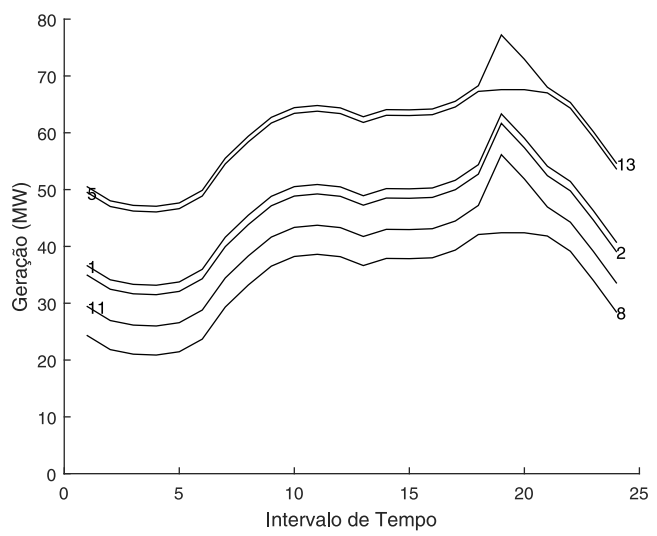

(d) Despacho com Reserva envolvendo os geradores 5 e 8.

Figura 1: Despachos com Manobras e Reserva Girante. 


\title{
6 CONCLUSÕES
}

Os estudos de casos mostram que é possível obter uma implementação eficiente de método de pontos interiores primal-dual para resolver o problema de pré-despacho com manobras programadas e reserva girante.

Com este estudo é possível observar que apenas manobras de linhas não provoca alterações no despacho caso não haja congestionamento nas demais linhas de transmissão. O tempo computacional para obter o despacho ótimo sofre uma leve alteração, mostrando que o custo computacional é relativamente o mesmo que sem manobras. O custo financeiro da operação aumenta, porém era de se esperar visto que o despacho ótimo considera as linhas que foram desligadas.

Ao inserir política de reserva girante observa-se que há alterações no despacho que podem variar conforme o conjunto de geradores escolhido. Para que haja uma menor alteração no despacho, e deste modo no custo computacional e operativo (considerando custos iguais), sugere-se escolher os geradores com menor demanda no despacho em operação normal.

Testes computacionais também foram realizados com problemas maiores como o Sistema IEEE118, Sul-Sudeste de 1654 barras e o Sistema Brasileiro. As observações foram semelhantes às obtidas com o Sistema IEEE-30.

Os resultados obtidos relacionadas ao pré-despacho confirmam a ideia que métodos de pontos interiores especializados a estruturas particulares levam a implementações que obtém bons resultados computacionais. A robustez obtida na implementação é um resultado esperado, típico destes métodos na área de sistemas de potência.

\section{AGRADECIMENTOS}

Agradecimento Auxílio a Fundação de Amparo à Pesquisa do Estado de São Paulo - FAPESP.

\begin{abstract}
In this paper the primal-dual interior point methods are developed for the problem of minimizing losses in the generation and transmission of the predispatch of a hydroelectric power system with programmed maneuvers and spinning reserve constraints. Both aspects, the number of iterations for the method to achieve convergence and the efficient solution of the resulting linear systems in order to obtain fast iterations are discussed.
\end{abstract}

Keywords: interior point, predispatch, linear programming.

\section{REFERÊNCIAS}

[1] E. Allen \& M. Ilic. Reserve markets for power systems reliability. IEEE Transactions on Power Systems, pages 228-233, (2000).

[2] S.M.S. Carvalho. Métodos de pontos interiores aplicados ao problema de pré-despacho de um sistema hidroelétrico com manobras programadas. Master's thesis, IMECC - UNICAMP, Agosto 2007. Dissertação de Mestrado. 
[3] C. Cavellucci. Sistema especialista em manobras de redes de distribuição de energia elétrica. Master's thesis, FEEC - UNICAMP, Junho 1989. Dissertação de Mestrado.

[4] M. Coelho, A. Oliveira \& S. Soares. Optimal power flow in DC constrained operating reserve through interior point methods. 2012 Sixth IEEE/PES Transmission and Distribution: Latin America Conference and Exposition (T\&D-LA), pages 1-5, (2012).

[5] Operador Nacional do Sistema Elétrico. Administração dos serviços ancilares. http://www.ons.org.br, (2007).

[6] Operador Nacional do Sistema Elétrico. Apuração dos serviços ancilares. http://www.ons.org.br, (2007).

[7] Operador Nacional do Sistema Elétrico. Arranjos comerciais para serviços ancilares prestados pelos agentes de operação. http://www.ons.org.br, (2007).

[8] F.P. Gomes J.C.F. Luz, M.Th. Schilling, R.N. Foutoura \& M. Groetaers. Sunsídios para distribuição de custos de serviços ancilares para gestão da rede. XIV SNPTEE, (1997).

[9] A.R.L. Oliveira \& S. Soares. Métodos de pontos interiores para problema de fluxo de potência ótimo DC. SBA: Controle \& Automação, 14(3) (2003), 278-285.

[10] A.R.L. Oliveira, S. Soares \& L. Nepomuceno. Optimal active power dispatch combining network flow and interior point approaches. Power Systems, IEEE Transactions on, 18(4) (2003), 1235-1240, Nov.

[11] A.R.L. Oliveira, S. Soares \& L. Nepomuceno. Short Term Hydroelectric Scheduling Combining Network Flow and Interior Point Approaches. Electrical Power \& Energy Systems, 27(2) (2005), 91-99.

[12] Y.M. Rodrigues \& O.R. Saavedra. Minimização dos custos do serviço de potência reativa fornecida pelos geradores com reserva de potência: Uma abordagem evolutiva. Revista Controle e Automação, pages 228-233, (2007).

[13] D. Shinmohommoadi \& A. Vojdani. An overview of ancillary services. V Simpósio de Especialistas em Planejamento Operacional de Expansão Elétrica, (2006).

[14] S. Soares \& C.T. Salmazo. Minimum loss predispatch model for hydroelectric systems. IEEE Transactions on Power Systems, 12(3) (1997), 1220-1228.

[15] R.J. Vanderbei. Linear Programming - Foundations and Extensions. Kluwer Academics Publishers, Boston, USA, (1996).

[16] S.J. Wright. Primal-Dual Interior-Point Methods. SIAM Publications, SIAM, Philadelphia, PA, USA, (1996). 